\title{
Gestión del conocimiento en la universidad: cuestionario para la evaluación institucional
}

Gabriela Cuadrado-Barreto

\section{RESUMEN}

La gestión del conocimiento (GC) en las universidades es un proceso cotidiano. Es importante detectar las características de la GC que se realiza en ellas. En este trabajo se plantea validar un cuestionario para evaluar la gestión de conocimiento de la universidad (CEGGU) por medio del juicio de expertos. La indagación es descriptiva, no experimental, transversal, se examinan los datos con alfa de Cronbach y análisis factorial exploratorio, se demuestra lo pertinente y comprensible del CEGCU. Se considera que éste es un instrumento que puede aportar el análisis del rendimiento organizacional de las universidades.

Palabras clave: cuestionario, gestión del conocimiento, capital intelectual, instituciones de educación superior, universidad.

\section{Gabriela Cuadrado-Barreto}




\section{Gestão do conhecimento na universidade: questionário para a avaliação institucional}

\section{RESUMO}

A gestão do conhecimento $(\mathrm{GC})$ nas universidades é um processo cotidiano. É importante detectar as características da GC que se realiza nelas. Neste trabalho se pretende validar um questionário para avaliar a gestão de conhecimento da universidade (GEGCU) por meio do juízo de especialistas. A indagação é descritiva, não experimental, transversal, examina-se os dados com alfa de Cronbach e análise fatorial exploratória, demostra-se o pertinente e compreensível do CEGCU. Se considera que este é um instrumento que possa aportar a análise do rendimento organizacional da universidade.

Palavras chave: questionário, gestão do conhecimento, capital intelectual, instituições de educação superior, universidade.

\section{Knowledge management at the university: questionnaire for institutional assessment}

\section{ABSTRACT}

Knowledge management $(\mathrm{KM})$ in universities is an everyday process. It is important to detect the characteristics of $\mathrm{KM}$ at universities. This article intends to validate a questionnaire drawn up to assess university knowledge management (Cuestionario para Evaluar la Gestión de Conocimientos de la Universidad, GEGGU) through its revision by experts. The inquiry is descriptive, non-experimental, transversal, and the data are examined by means of Cronbach alpha and exploratory factorial analysis, in order to prove the relevance and comprehensibility of the CEGCU, that can be considered an instrument able to provide the analysis of the organizational performance of the university.

Key words: questionnaire, knowledge management, intellectual capital, higher education institutions, University. 


\section{Introducción}

La evaluación organizacional de la universidad latinoamericana parte de la función social que, con propósitos de mejora, se lleva a cabo en las instituciones universitarias. $\mathrm{Al}$ considerar que en la evaluación de la universidad se consideran indicadores internacionales de indagación de resultados de conocimiento (Moreno y Ruiz, 2009), es necesario evaluar la gestión de los activos intelectuales a partir de la importancia que otorga la sociedad al desarrollo de conocimiento especializado proveniente de las instituciones de educación superior (IESALC, 2007). Derivado de ello, el ejercicio de las funciones sustantivas de la universidad vuelca sus esfuerzos en la creación, transferencia, almacenamiento, aplicación y uso del conocimiento - como procesos particulares de gestión del conocimiento $(\mathrm{GC})$ - , por lo que es esencial contar con instrumentos de diagnóstico que integren tanto los procesos de GC, como los elementos del capital intelectual (CI) - capital humano, capital estructural y capital relacional- en el marco universitario. En este trabajo, con base en la teoría administrativa, se plantea la metodología para la indagación; a continuación, con base en la creación del conocimiento, recursos y capacidades organizacionales, se esboza el marco teórico que lleva a la creación de un Cuestionario para la evaluación de la gestión del conocimiento universitario (CEGCU); y las pautas para validar su eficiencia.

\section{Perspectiva metodológica y método de investigación}

El considerar a la organización universitaria como un sistema complejo implica, ineludiblemente, la necesidad de profundizar tanto en los procesos de gestión que ejecuta como en los resultados organizacionales que obtiene. El objeto de estudio complejo de la investigación se denomina Cuestionario para la evaluación de la gestión del conocimiento universitario (CEG$C U)$, que integra los procesos particulares de GC y de los elementos del CI a través de criterios de análisis, escalas e indicadores para evaluar a la organización universitaria. La validación del CEGCU se realiza a través del juicio de nueve expertos investigadores de las áreas de gestión del conocimiento, capital intelectual, universidad, evaluación de la organización universitaria. A su vez, los expertos validan el CEGCU en lo pertinente y lo comprensible de los 80 ítems que lo constituyen por medio de un cuestionario digital autoadministrado (GoogleDocs) que integra una escala diferencial semántica - entre uno y cinco- (Vaca, 2015). El análisis de los datos obtenidos se realiza por medio del análisis factorial exploratorio, así como de los coeficientes Alfa de Cronbach y Káiser-Meyer-Olkin (KMO). Además, se presenta el CEGCU como referente teórico válido en las conclusiones de la investigación.

\section{Los procesos de gestión del conocimiento en la organización universitaria}

La revisión teórico-conceptual inicia con la asunción del conocimiento organizacional como una variable de contingencia generado mediante procesos particulares de gestión, enmarcados en un contexto determinado y que impulsa a obtener resultados institucionales de activos de conocimiento. De esta forma, el proceso de $\mathrm{GG}$ - calificado como continuo y dinámico-, identifica y muestra el conocimiento, además de incidir en el desempeño organizacional. Derivado de ello, la evaluación del proceso de GC se define como un mecanismo capaz de identificar y medir el desempeño organizacional por medio de herramientas (Birkinshaw, Nobel y Ridderstrale, 2002; Von Krogh, Nonaka y Rechsteiner, 2012; Lupton y Beamish, 2014; Markova y Ford, 2011; Kaziliünas, 2012). Entonces, la GC se describe como un proceso organizacional que, al integrar procesos particulares, requiere ser evaluado y medido.

La organización universitaria, al poseer el contexto propicio para la interacción entre sus integrantes - docentes, investigadores, estudiantes y 
empleados - genera de forma continua conocimiento científico y tecnológico de importancia para la sociedad (Misiūnaitė y Jucevičienè, 2014). De esta forma, se identifican los procesos particulares de creación (CC), transferencia/almacenamiento (TA) y aplicación/uso del conocimiento (AU) que integran la GC universitario; estos procesos interaccionan de acuerdo con las funciones efectuadas por las organizaciones universitarias, tales como enseñanza, investigación, vinculación con la sociedad y difusión de la cultura (Cuadrado y Carrillo, 2016).

La creación de conocimientos (CC) se reconoce como un proceso social, desarrollado a través de la interacción entre individuos en un contexto físico o virtual, que promueve el desarrollo de diferentes formas de conocimiento (Konno, Nonaka y Ogilvy, 2014 a, b, c), involucra la adquisición, diseminación e interpretación de la información que, a su vez, genera la transformación del conocimiento organizacional - tácito en explícito-. La transferencia de conocimiento en la organización universitaria promueve - de manera física o por medio de la red informática - la distribución de propuestas, el ejercicio de mejores prácticas, el desarrollo de habilidades y la adquisición de nuevos conocimientos que incentiven el desarrollo organizacional (Alegre, Sengupta y Lapiedra, 2011). El almacenamiento del conocimiento, a través de las bases de datos desarrolladas con tecnologías de la información, permite la recolección de propuestas, el almacenaje de experiencias, el manejo de información compartida y la rotación de personal para evitar pérdidas de conocimiento, impedimentos de acceso a éste de forma libre y adecuada (Wardini, 2015; Demchig, 2015). De esta forma, la transferencia/almacenamiento de conocimiento (TA) se configura como proceso interdependiente y vinculante en su propia especificidad funcional, considerado como sostén del desarrollo de las funciones ejecutadas por los integrantes de la universidad que apoya a los procesos de CG y AU. La aplicación del conocimiento se ejecuta a través del desarrollo de las funciones universitarias y el uso del conocimiento se genera como parte de la búsqueda de soluciones de los trabajadores universitarios a los problemas que se presentan en la frontera de la ciencia del conocimiento en la organización académica (Badah, 2012) $\mathrm{y}$, con base en lo anterior, AU se construye como un proceso particular interdependiente y vinculante. De esta forma, la interacción entre los procesos particulares de creación, transferencia/almacenamiento y aplicación/uso del conocimiento permite definir a la organización universitaria como un sistema complejo que integra diversos elementos para el cumplimiento de sus funciones sociales (véase figura 1). 
Figura 1. Proceso particular de gestión del conocimiento universitario

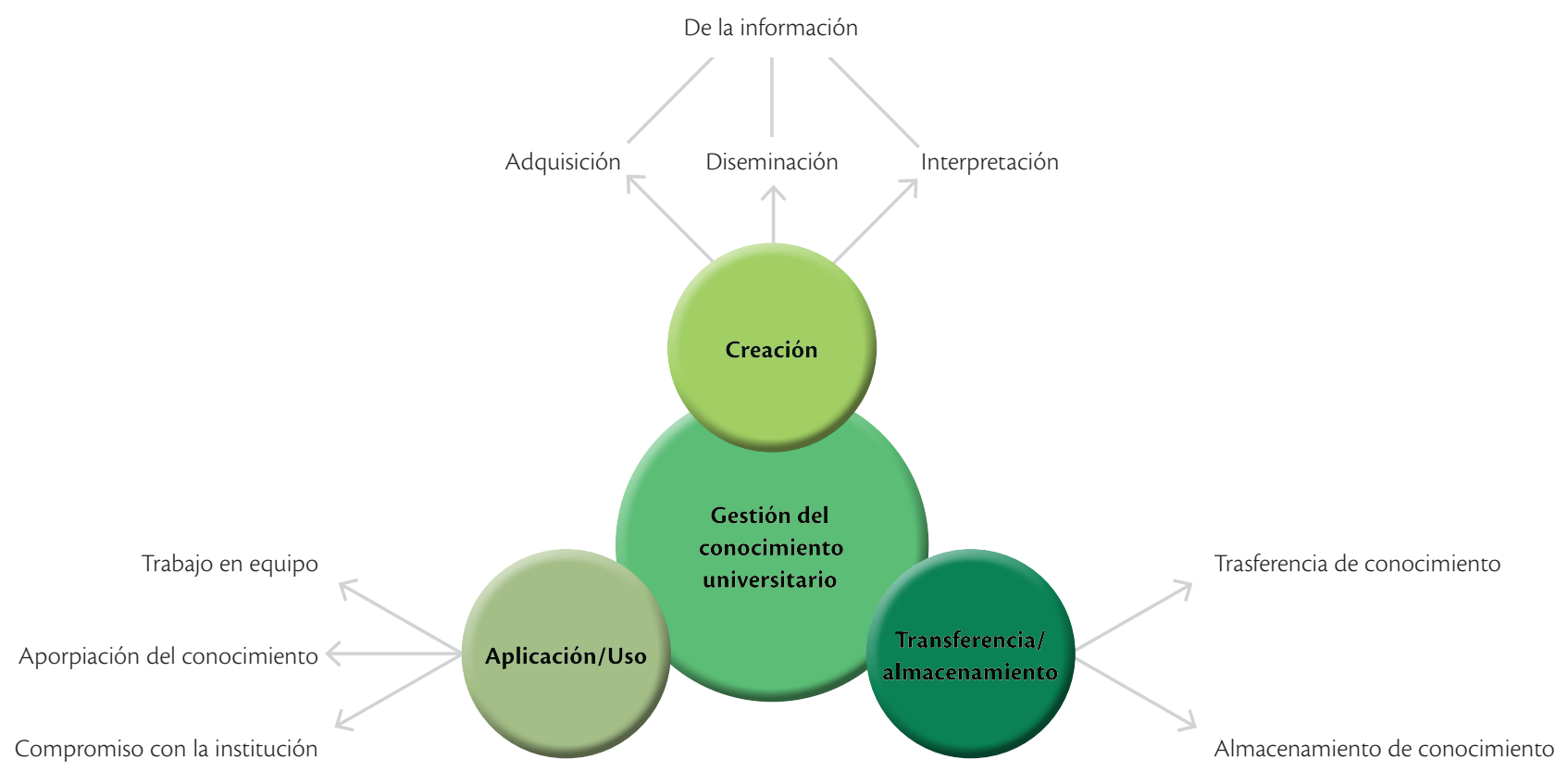

Fuente: elaboración propia.

\section{Elementos del capital intelectual universitario}

La importancia social de la universidad radica en su producción de conocimiento científico y tecnológico, mismo que incentiva el crecimiento económico del entorno en el que la entidad universitaria se ubica (Brunner y Ferrada, 2011). El conocimiento se identifica, entonces, como un activo intangible cuya vinculación con los activos tangibles de la organización produce resultados de conocimiento denominados capital intelectual (Barney, 2001; Martín, 2014). Para la presente investigación, el CI universitario se define como los resultados de conocimiento obtenidos a partir de la ejecución de procesos organizacionales y se construye a partir de tres elementos sustantivos: capital humano $(\mathrm{CH})$, capital estructural $(\mathrm{CE})$ y capital relacional (CR), clasificados en función de características identificables y controlables, propias de los activos intangibles, para definir la estructura organizacional de la universidad (Edvinsson y Malone,
1999; Bueno, Rodríguez y Salmador, 1999; Carrillo, 2010; NIC-38, 2013).

El CH combina los conocimientos, habilidades, inventiva, capacidad, experiencia, innovación y creatividad de docentes e investigadores con los valores, la cultura y la filosofía organizacional para la realización del trabajo asignado (Ramírez y Gordillo, 2014). Muestra, igualmente, que la participación de los docentes en proyectos de investigación internacional, las visitas/estancias internacionales y las delegaciones de alta responsabilidad incrementan el conocimiento, las destrezas y habilidades de los docentes (Nava, Mercado y Demuner, 2012; Ramírez, Tejada y Gordillo, 2013). La experiencia de los maestros se incrementa en función del tiempo de ejercicio de su cátedra, así como de las estancias de investigación en el extranjero (Karami y Vafaei, 2014). Además, la innovación y creatividad se explicitan a través de patentes, derechos de propiedad intelectual y de autor, publicaciones científicas, 
capítulos de libros, memorias de congresos, citas y visibilidad de sitios web (Aerden, 2014; Ramírez, Tejada y Baidez, 2013).

El CE refiere a la infraestructura física propiedad de la universidad, misma que facilita el acceso al conocimiento, por lo que es independiente de las personas y se evidencia como activos organizacionales, muebles e inmuebles (Lu, Wang y Kweh, 2014). Los sistemas informáticos son herramientas tecnológicas de apoyo a los procesos de GC; la cultura organizacional une costumbres y normas establecidas por la dirección universitaria; la estructura organizacional facilita la comunicación interjerárquica y el ejercicio de los derechos de propiedad intelectual para proteger los activos de conocimiento (Najim, Al-Naimi y Alnaji, 2012; Nonaka y Takeuchi, 1995; Nava y Mercado, 2011). De esta manera, el CE se considera la base de la producción de los integrantes de la organización.

El CR aúna el conjunto de relaciones económicas, políticas e institucionales desarrolladas por la universidad a través del tiempo (Ramírez, Santos y Tejada,
2012). Integra a los proveedores como personas externas a la organización y se vincula con la sociedad para promover la difusión del conocimiento, la reputación institucional generada a través de acreditaciones y/o evaluaciones, y las redes de trabajo conformadas para favorecer la ejecución de las funciones universitarias sustantivas (Houston y Paewai, 2013; Kenway y Fahey, 2014).

Así, los tres elementos del capital intelectual - capital humano $(\mathrm{CH})$, capital estructural $(\mathrm{CE})$ y capital relacional (CR) - interactúan de forma interdependiente por medio de las características de sistema complejo de la universidad, al mismo tiempo que cumplen funciones específicas. El CH interrelaciona el conocimiento tácito y explícito para proporcionar conocimiento científico y tecnológico a la universidad; el CE coloca al alcance de los integrantes de la comunidad universitaria el conocimiento desarrollado $y$, finalmente, el CR, permite el flujo de conocimiento desde el entorno externo de la organización hacia el interior de la misma y viceversa (véase figura 2).

\section{Figura 2. Elementos del capital intelectual universitario}

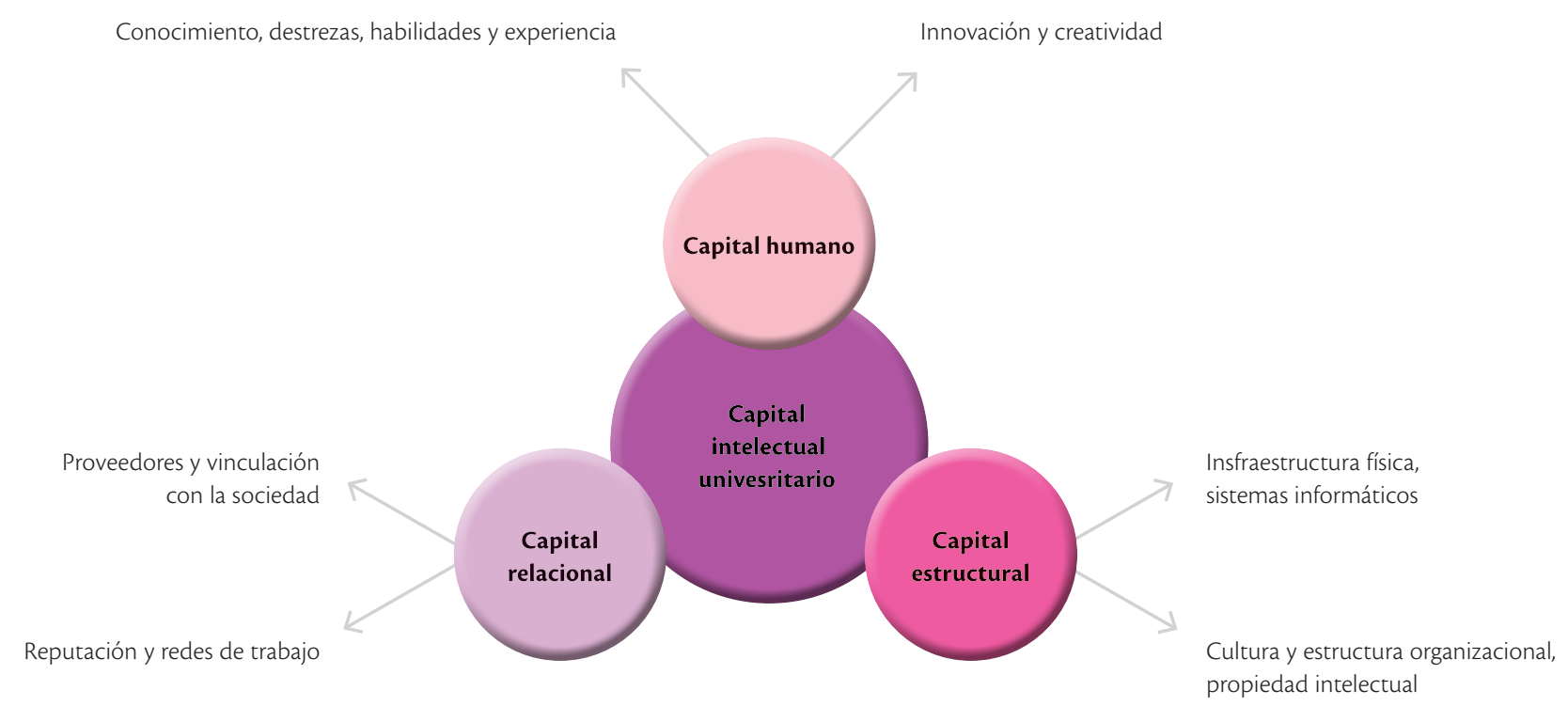




\section{Evaluación de la organización universitaria}

Los cuestionarios de evaluación de la organización universitaria son de utilidad para medir y comparar el rendimiento institucional de forma cuantitativa (IREG, 2015), sin embargo, debido a las particularidades involucradas en la ejecución de los procesos de gestión institucional, la evaluación de estos procesos quedan fuera de su alcance.

La universidad ejecuta las funciones de enseñanza, investigación, vinculación social y difusión de la cultura, basadas en la GC que desarrolla, mismas que generan beneficios a la sociedad (United Nations, 2005; World Bank, 2013). La incidencia del conocimiento universitario en el desempeño organizacional lo convierte en un elemento sustantivo de las instituciones de educación superior (IES), y por ende, primordial a la hora de definir la gestión del conocimiento como un proceso social continuo y dinámico; además, identificar y mostrar el capital intelectual de la universidad parte de la ejecución de las funciones de la organización (Ramírez y Gordillo, 2014). Al respecto, la literatura es diversa en cuanto al desarrollo y validación de instrumentos, por una parte, de evaluación de la GC universitaria, por otra parte, del CI universitario y son varios los ejemplos de instrumentos de medición enfocados a tratar de evaluar la GG y el CI (véase tabla 1).

Sin embargo, ninguno de los ejemplos mencionados recoge de forma simultánea información sobre la ejecución de los procesos de GC y los resultados de CI de la organización universitaria, tal y como sucede con el CEGCU.

Tabla 1. Instrumentos de identificación de la GC y el cl en el ámbito de la educación superior

\begin{tabular}{|c|c|c|}
\hline Autor & Descripción & Propósito \\
\hline Bueno et al. (2003) & Relación entre los procesos de cc con los elementos del Cl. & $\begin{array}{l}\text { Reflejar los procesos de cc en los resultados de } \\
\text { producción científica de las universidades. }\end{array}$ \\
\hline Sánchez, Castrillo y Elena (2006) & $\begin{array}{l}\text { Diferencia los constructos de CH, CE y CR como financieros y } \\
\text { no financieros. }\end{array}$ & $\begin{array}{l}\text { Presentar el cuestionario "The ICU report" en el } \\
\text { ámbito de las universidades españolas. }\end{array}$ \\
\hline Bustillos (2012) & Presenta la GC a través de los elementos del $\mathrm{Cl}$. & $\begin{array}{l}\text { Promover la productividad académica de los } \\
\text { institutos tecnológicos en México. }\end{array}$ \\
\hline Bucheli et al. (2012) & $\begin{array}{l}\text { Muestra la producción de conocimiento de las universidades } \\
\text { colombianas a largo plazo en términos de } \mathrm{Cl} \text {. }\end{array}$ & $\begin{array}{l}\text { Determinar la acumulación del conocimiento de las } \\
\text { universidades a través del tiempo. }\end{array}$ \\
\hline $\begin{array}{l}\text { Ali Siadat, Hoveida, Abbaszadeh y } \\
\text { Moghtadaie (2012) }\end{array}$ & $\begin{array}{l}\text { Relación entre las escalas de adquisición, diseminación e } \\
\text { interpretación de la información. }\end{array}$ & $\begin{array}{l}\text { Reconocer el proceso particular de creación de } \\
\text { conocimiento en universidades de Irán. }\end{array}$ \\
\hline Salleh (2014) & $\begin{array}{l}\text { Propuesta de un cuestionario del proceso de gestión del } \\
\text { conocimiento tácito. }\end{array}$ & $\begin{array}{l}\text { Reconocer el proceso de transferencia de } \\
\text { conocimiento de las universidades públicas de } \\
\text { Malasia. }\end{array}$ \\
\hline Chong, Yuen y Gan (2014) & $\begin{array}{l}\text { Propuesta de un cuestionario de intercambio de } \\
\text { conocimiento entre el personal académico. }\end{array}$ & $\begin{array}{l}\text { Examinar las percepciones del proceso particular de } \\
\text { transferencia del conocimiento en las universidades } \\
\text { de Malasia. }\end{array}$ \\
\hline Ordorika (2014) & $\begin{array}{l}\text { Integra el cı con indicadores nacionales de evaluación } \\
\text { universitaria en México. }\end{array}$ & $\begin{array}{l}\text { Presentar los activos universitarios del } \\
\text { conocimiento. }\end{array}$ \\
\hline Mercado, Gil y Demuner (2014) & $\begin{array}{l}\text { Estructura el } \mathrm{Cl} \text { a partir de tres elementos: capital humano } \\
(\mathrm{CH}) \text {, capital estructural (CE) y capital relacional (CR). }\end{array}$ & $\begin{array}{l}\text { Evaluar los resultados de conocimiento de las } \\
\text { universidades mexicanas. }\end{array}$ \\
\hline Ramírez y Manzaneque (2015) & Integran los elementos CH, CE y CR. & $\begin{array}{l}\text { Identificar la importancia otorgada por los } \\
\text { integrantes de las universidades españolas a los } \\
\text { resultados organizacionales. }\end{array}$ \\
\hline
\end{tabular}

Fuente: elaboración propia basada en los autores citados. 


\section{Validación del Cuestionario de evaluación de la gestión del conocimiento de la universidad (CEGCU) mediante juicio de expertos}

Creado específicamente para la investigación que aquí se presenta, el CEGGU, se perfila como un instrumento relevante en la recolección de información asociada a los procesos de gestión del conocimiento efectuados por universidad y al capital intelectual desarrollado por la misma en un periodo determinado de tiempo. El propósito del instrumento es evaluar la GC a través de las percepciones de los directores de carrera sobre los procesos de creación (CG), transferencia/ almacenamiento (TA) y aplicación/uso (AU) de conocimiento, efectuados por los integrantes de la organización como parte de sus actividades diarias.

El CEGCU se construyó a partir de dos tipos de reactivos - ítems ya ensayados para aumentar su validez, e ítems desarrollados de manera específica fundamentados en la teoría previamente descrita-, y fue sometido al juicio de investigadores expertos en el área de estudio para su validación. La medición de la variable GC se efectuó a partir de una escala diferencial semántica de cinco posiciones, ${ }^{1}$ que se considera una herramienta adecuada para extraer datos cuantitativos y permite aplicar un tratamiento estadístico a la variable (Che Rusuli, Tasmin, Takala y Hashim, 2014). La medición de la variable CI se llevó a cabo a través de un cuestionamiento sobre el número real de productos académicos que pueden identificarse y cuantificarse en un periodo de tiempo determinado (Mercado, Sánchez y Sánchez, 2013). Los ítems se formularon de forma clara, precisa y léxicamente sencilla; se descartaron formulaciones estereotipadas o sesgadas que pudieran causar reacciones imprevistas, - negativas o positivas - en el informante; el formato se consideró amable - para la lectura y la cumplimentación manual o electrónica - con un diseño factible para la autoadministración - indicaciones para el llenado - y la obtención de información adicional relevante para el estudio.

El CEGCU, como instrumento de evaluación organizacional para el monitoreo de los procesos particulares de GC universitario, se construyó por medio de la identificación y definición de las variables de GC y CI, las dimensiones (creación de conocimiento, transferencia/ almacenamiento de conocimiento, aplicación/ uso de conocimiento, capital humano, capital estructural y capital relacional), los indicadores (80), y se dirigió a los directores de carrera como informantes de calidad. El cuestionario integra tres partes (véase anexo 1), la primera parte recopila información sociodemográfica sobre la institución a evaluar y sus informantes. La segunda parte - sección 1 del CEG$\mathrm{CU}$ - conformada por ítems relacionados con la GC - 41 ítems - , se sustenta sobre una escala diferencial semántica de cinco niveles - de 1 a 5- que se corresponden con los niveles de procesos de gestión ejecutados por las universidades (Cordero, 2015). ${ }^{2}$ La tercera parte - sección 2 del CEGCU - se constituye por los ítems correspondientes al CI — 39 ítems - donde se solicita a los informantes respuestas cuantitativas concretas - cifras - en relación con los productos de conocimiento desarrollados por la organización en el plazo de un año. Los resultados extraídos se equiparan para su procesamiento con la escala diferencial semántica antes descrita, con el fin de determinar la producción de conocimiento universitario. ${ }^{3}$

\footnotetext{
${ }^{1}$ Se pide a los expertos que valoren las afirmaciones con una escala de diferencial semántica de 5 puntos, 1 significancia que los procesos son desorganizados y 5 que se han depurado hasta un nivel de mejor práctica.

${ }^{2}$ El nivel 1 o inicial indica que el proceso se desarrolla de manera desorganizada; el nivel 2 o repetible, alude a un patrón regular de ejecución del proceso; el nivel 3 o definido, remite a un proceso estandarizado en la organización; el nivel 4 o administrado, señala la integración y monitoreo del proceso en la universidad; finalmente, el nivel 5 u optimizado, indica que el proceso de GG se ha depurado hasta alcanzar las mejores prácticas. ${ }^{3}$ El nivel inicial muestra los activos de conocimiento en una escala entre uno (1) y diez (10): en el nivel de patrón regular se recogen los activos intangibles en una escala de once (11) a veinte (20); el nivel estandarizado expone los resultados en una escala de veintiuno (21) a treinta (30); el nivel administrado ubica los datos obtenidos en una escala de treinta y uno (31) a cuarenta (40) y, finalmente, el nivel optimizado, da a conocer que los activos de conocimiento alcanzan en la escala un puntaje de cuarenta y uno (41) o superior.
} 
La evaluación del sistema complejo CEGGU corresponde a la validación del instrumento mediante el juicio de expertos y al análisis estadístico de los resultados de la validación.

$\mathrm{Al}$ considerar que la universidad, como centro generador de conocimiento dentro de la sociedad, se encuentra integrada por personas que realizan actividades para el cumplimiento de las funciones organizacionales (Wigmore y Ruiz, 2014), la selección de los expertos, a cuyo juicio se sometió el CEGCU, se efectuó con base en dos criterios: la caracterización, es decir, el investigador consultado mantiene líneas de investigación homogéneas que incluyan gestión del conocimiento, capital intelectual y universidad; y, en segundo término, la procedencia del experto, ya que con el propósito de asegurar su conocimiento de la realidad universitaria, se requiere un mínimo de cuatro años de trabajo en instituciones educación superior como docente investigador (Mochales, 2014).

A partir de los criterios citados, fueron considerados nueve expertos en las áreas de gestión del conocimiento, capital intelectual y evaluación de organizaciones universitarias, pertenecientes a universidades públicas, privadas y a organismos estatales de evaluación universitaria, cuya diversidad de perspectivas garantiza una validación de amplio espectro y, por ende, un aporte de relevancia notable en el contexto universitario latinoamericano. De esta manera, la obtención de datos empíricos otorgados por los expertos en universidades permitió la validación del cuestionario CEGCU.

La validación del cuestionario mediante el juicio de expertos se realizó en función de la necesidad de que todo instrumento de medición precisa de la opinión informada de personas con amplia trayectoria en la materia objeto de estudio y sólida reputación profesional, capaces de proporcionar información, evidencia, juicios y valoraciones a los elementos del instrumento (Escobar y Cuervo, 2008), contar con disponibilidad, motivación para participar en el estudio y con la voluntad de emitir una opinión imparcial (Skjong y Wentworht, 2000). La validación, en lo pertinente y lo comprensible de todos y cada uno de sus ítems, se efectuó de manera individual mediante la técnica de acuerdo entre codificadores (Vaca, 2015), y una escala diferencial semántica de cinco niveles - total desacuerdo, en desacuerdo, parcialmente de acuerdo, de acuerdo y total acuerdo-, cuyos resultados se sistematizaron como sigue: Total desacuerdo y en desacuerdo, se equipararon a una negación - $\mathrm{NO}$-; de acuerdo y total acuerdo, a una aceptación - Sí-; por último, parcialmente, se equiparó con indecisión.

$\mathrm{El}$ análisis de la consistencia interna del CEGCU se basó en el promedio de las correlaciones entre los ítems por medio del coeficiente Alfa de Cronbach, lo que permitió evaluar cuánto mejoraría - o empeoraría - la fiabilidad de la prueba si se excluyera determinado ítem (García-Bellido et al., 2010). En el software estadístico SPSS V. 20 se efectuó el análisis estadístico del coeficiente Alfa de Cronbach de las dimensiones y los criterios del cuestionario, especificando que aquellos elementos con un valor de Alfa de Cronbach menor a 0.7 como valor de corte, serían excluidos de la versión final. Finalmente, tras analizar los 80 ítems que constituyen el CEGCU, los expertos concluyeron que éste era pertinente, en su conjunto, con lo propuesto desde la teoría en un 0.92 y en 0.91, en relación con la comprensibilidad del conjunto de los ítems (véanse tablas 2 y 3 ).

El ordenamiento general de los criterios que componen el CEGCU según su nivel de importancia, permite afirmar que los expertos concedieron mayor relevancia a elementos del capital humano, capital estructural y capital relacional, como son la experiencia internacional, la innovación y la creatividad reflejada en la publicación de productos de investigación, la infraestructura física de la organización para apoyo de las actividades docentes, la cultura organizacional y la propiedad intelectual universitaria, así como a las redes de trabajo internas y externas de las que forma parte la institución (véase tabla 3). 
Gestión del conocimiento en la universidad: cuestionario para la evaluación institucional

Tabla 2. Validación de los criterios del CEGCU

\begin{tabular}{|c|c|c|c|}
\hline \multirow{2}{*}{ Criterios } & \multirow{2}{*}{ Núm. de ítems } & \multicolumn{2}{|c|}{ Alfa de Cronbach } \\
\hline & & Pertinente & Comprensible \\
\hline Creación de conocimiento & 14 & 0.88 & 0.80 \\
\hline Transferencia-almacenamiento de conocimiento & 11 & 0.77 & 0.85 \\
\hline Aplicación-uso de conocimiento & 16 & 0.80 & 0.75 \\
\hline Capital humano & 16 & 0.78 & 0.88 \\
\hline Capital estructural & 12 & 0.87 & 0.77 \\
\hline Capital relacional & 11 & 0.86 & 0.87 \\
\hline
\end{tabular}

Fuente: elaboración propia.

Tabla 3. Validación de las escalas del CEGCU

\begin{tabular}{|c|c|c|c|}
\hline \multirow{2}{*}{ Criterios } & \multirow{2}{*}{ Núm. de ítems } & \multicolumn{2}{|c|}{ Alfa de Cronbach } \\
\hline & & Pertinente & Comprensible \\
\hline \multicolumn{4}{|c|}{ Creación de conocimiento } \\
\hline Adquisición de la información & 7 & 0.70 & 0.69 \\
\hline Diseminación, interpretación de la información & 7 & 0.72 & 0.71 \\
\hline \multicolumn{4}{|c|}{ Transferencia/almacenamiento de conocimiento } \\
\hline Almacenamiento de conocimiento & 5 & 0.69 & 0.71 \\
\hline Transferencia de conocimiento & 6 & 0.70 & 0.68 \\
\hline \multicolumn{4}{|c|}{ Aplicación/uso de conocimiento } \\
\hline Trabajo en equipo & 5 & 0.71 & 0.69 \\
\hline Apropiación del conocimiento & 5 & 0.70 & 0.70 \\
\hline Compromiso con el conocimiento & 6 & 0.68 & 0.72 \\
\hline \multicolumn{4}{|c|}{ Capital humano } \\
\hline Conocimiento, destrezas, habilidades y experiencia & 6 & 0.68 & 0.70 \\
\hline Innovación y creatividad & 10 & 0.71 & 0.67 \\
\hline \multicolumn{4}{|c|}{ Capital estructural } \\
\hline Infraestructura física, sistemas informáticos & 6 & 0.70 & 0.72 \\
\hline Cultura y estructura organizacional, propiedad intelectual & 6 & 0.71 & 0.67 \\
\hline \multicolumn{4}{|c|}{ Capital relacional } \\
\hline Proveedores y vinculación con la sociedad & 5 & 0.71 & 0.69 \\
\hline Reputación y redes de trabajo & 6 & 0.70 & 0.71 \\
\hline
\end{tabular}

Fuente: elaboración propia. 
Igualmente, por sugerencia de los expertos, se reelaboraron varios ítems con propósitos de optimizar su legibilidad. El análisis factorial exploratorio expuso que el coeficiente Káiser-Meyer-Olkin (KMO) presentó un valor igual a 0.82; la prueba de Bartlett fue también significativa $(\mathrm{P}<0,01)$ y, de esta manera, pudo concluirse que existe un alto grado de interrelación entre las variables de GC y CI en los seis componentes principales del CEGCU, que se muestra plenamente validado al agrupar claramente a sus ítems.

\section{Discusión y conclusiones}

$\mathrm{Al}$ considerar la importancia del conocimiento que generan las universidades para el desarrollo de las naciones, la presente investigación propone la validación del Cuestionario para la evaluación de la gestión del conocimiento de la universidad. A través de la indagación de lo pertinente y lo comprensibles, de los ítems que integran el CEGCU los expertos manifiestan que las escalas creación del conocimiento, capital estructural y capital relacional tienen gran importancia para la evaluación de la institución universitaria, lo que coincide con investigaciones internacionales como las de Mercado, Gil y Demuner (2014), IREG (2015). Además, el capital humano, la aplicación, uso, transferencia y almacenamiento de conocimiento se muestran como importantes al manifestar los propósitos de la institución, tales como la enseñanza o la investigación, por lo que el CEGCU permite la comparación entre las organizaciones de educación superior.

Tras lo expuesto previamente, puede concluirse que el CEGCU se diferencia de otros instrumentos de diagnóstico de la gestión del conocimiento en el hecho de recolectar información tanto de los procesos organizacionales como de los resultados de conocimiento, ofreciendo una visión más clara y precisa de la ejecución de las funciones de la entidad universitaria. Así mismo, permite un análisis detallado de todos y cada uno de los procesos de GG y de los elementos del capital intelectual a través de los criterios, las escalas y los ítems que lo conforman, presentando a la organización como un todo.

El CEGCU incluye tres procesos particulares de GC y tres elementos de CI - creación, transferencia/ almacenamiento, aplicación/uso de conocimiento, capital humano, capital estructural y capital relacional-, mismos que fueron desprendidos de una profunda revisión literaria, lo que aunado a los resultados obtenidos en su validación, permite concluir que puede ser generalizado sin dificultad para su aplicación a toda institución de educación superior que requiera ser evaluada, independientemente de su naturaleza pública, privada o mixta. El CEGCU se define como una avanzada herramienta para la evaluación de la GC que muestra los resultados organizacionales de las universidades. También, el cuestionario permite extraer los datos necesarios para efectuar análisis estadísticos de la universidad en su conjunto y de las unidades académicas - facultades y direcciones de carrera - de forma individual, así como establecer comparaciones con otras organizaciones similares. Además, admite la localización de los procesos particulares de GC que requieren mayor énfasis en su ejecución para, de esta manera, mejorar los resultados organizacionales expresados en capital intelectual. Asimismo, posibilita el monitoreo del desarrollo de las funciones sustantivas de la universidad - enseñanza, vinculación con la sociedad, investigación y difusión de la cultura - y, por ende, permite comparar los resultados de las mismas a lo largo del tiempo. Para investigaciones futuras se recomienda implementar un programa informático que permita la aplicación del GEGCU de forma simultánea en varias organizaciones universitarias, el procesamiento de los datos y la comparabilidad entre las instituciones participantes. 


\section{Referencias}

Aerden, Axel (2014), "Joint programme checklist: inspired by quality assurance, European Consortium for Accreditation in higher education", European Consortium for Accreditation in higher education (ECA), The Hague, The Netherlands, European Commission - Erasmus Mundus, pp. 32.

Alegre, Joaquín, Kishore Sengupta y Rafael Lapiedra (2011), "Knowledge management and innovation performance in a high-tech SMEs industry", International Small Business Fournal, vol. 31, issue 4, pp. 454-470 [DOI: 10.1177/0266242611417472].

Ali Siadat, Sayed, Reza Hoveida, Mohammad Abbaszadeh y Leila Moghtadaie (2012), "Knowledge creation in universities and some related factors", Journal of Management Development, vol. 31, issue 8, pp. 845-872.

Badah, Ahmad (2012), "Relationship between the knowledge management processes and the administrative empowerment with the employees of the Ministry of Higher Education and Scientific Research-Jordan", European Scientific Journal, vol. 8, núm. 28, pp. 191-209.

Barney, Jay (2001), "Resource-based theories of competitive advantage: A ten year retrospective on the resource-based view", fournal of management, vol. 27, Issue 6. pp. 643-650.

Birkinshaw Julian, Robert Nobel y Jonas Ridderstrale (2002), "Knowledge as a contingency variable: do the characteristics of knowledge predict organization structure?", Organization Science, vol. 13, issue 3, pp. 274289.

Brunner, José Joaquín y Rocío Ferrada Hurtado (eds.) (2011), Educación Superior en Iberoamérica: Informe 2011, Santiago de Chile, Banco Mundial/Centro Interuniversitario de Desarrollo (CINDA)/Universia.

Bucheli, Victor, Adriana Díaz, Juan Pablo Calderón, Pablo Lemoine, Juan Alejandro Valdivia, José Luis Villaveces y Roberto Zarama (2012), "Growth of scientific production in Colombian universities: an intellectual capital-based approach", Scientometrics, vol. 91, issue 2, pp. 369-382.

Bueno, Eduardo, Pilar Rodríguez y Maria Paz Salmador (1999), Experiencias en medición de capital intelectual en España: El Modelo Intellect, IX Congreso Nacional de Acede, pp. 26.

Bueno, Eduardo, Patricio Morcillo, Jesús Rodríguez, María Ángeles Luque, Mercedes Cervera, Claudia Camacho, Belén Merino, Cecilia Rivera, Oscar Rodríguez, Julián Villanueva y Lidia Villar (2003), Gestión del conocimiento en universidades y organismos públicos de investigación, Madrid, Dirección General de Investigación, Consejería de Educación, Comunidad de Madrid.

Bustillos, Elva (2012), "La gestión del conocimiento para promover la productividad académica de los institutos tecnológicos en la sociedad del conocimiento", Tesis Doctoral, México, Instituto Politécnico Nacional, ESCA. Carrillo Velázquez, Lucia Patricia (2010), "Knowledge management process and technology capacity in a social sciences network research", The fournal of Knowledge Economy \& Knowledge Management, vol. V, pp. 153-164.

Che Rusuli, Muhamad Saufi, Rosmaini Tasmin, Josu Takala y Norazlin Hashim (2014), "A structural equation model of knowledge management practices and library users' satisfaction at malaysian university libraries", The 8th International Conference on Knowledge Management in Organizations, Kaoshi ung, Taiwan, pp. 139-153.

Chong Chin, Wei, Yee Yen Yuen y Chew Gan Geok (2014), "Knowledge sharing of academic staff", Library Review, vol. 63, issue 3, pp. 203-223.

Cordero, Diego (2015), "Modelo para gobierno de tecnologías de la información (GTI): caso de las universidades cofinanciadas de la zona 6 de la República del Ecuador", México, Ponencia presentada en el Coloquio Doctoral, Universidad Nacional Autónoma de México.

Cuadrado, Gabriela y Patricia Carrillo (2016), "Los procesos de gestión del conocimiento. El caso de las universidades del Ecuador", XX Congreso internacional de investigación en ciencias administrativas (ACACIA), Merida, 
Yucatán, Universidad Autónoma de Yucatán.

Demchig, Bolormaa (2015), "Knowledge management capability level assessment of the higher education institutions: Case study from Mongolia", Procedia Social and Behavioral Sciences, vol. 174, pp. 3633-3640.

Edvinsson, Leif y Michael Malone (1999), El capital intelectual: cómo identificar y calcular el valor de los recursos intangibles de su empresa, Barcelona, España, Ediciones Gestión 2000.

Escobar-Pérez, Jazmine y Ángela Cuervo-Martínez (2008), "Validez de contenido y juicio de expertos: una aproximación a su utilización”, Avances en Medición, vol. 6, pp. 27-36.

García-Bellido, Rosario, José González Such y Jesús Jornet Melia (2010), Análisis de fiabilidad. Alpha de Cronbach, <http://www.uv.es/innomide/spss/SPSS/ SPSS_0801B.pdf> [Consulta: abril de 2016].

Houston, Don y Shelley Paewai (2013), "Knowledge, power and meanings shaping quality assurance in higher education: a systemic critique", Quality in Higher Education, vol. 19, issue 3, pp. 261-282.

Instituto Internacional para la Educación Superior en América Latina y el Caribe (IESALC) (2007), El compromiso social de las universidades de América Latina y el Caribe, Belo Horizonte, Brasil, IESALC.

IREG-Observatory on academic ranking and excellence (2015), Guidelines for stakeholders of academic rankings, $<$ http://ireg-observatory.org/en/index.php/ initiatives $>$ [Consulta: agosto de 2017].

Karami, Siavash y Alireza Vafaei (2014), "Australian universities and intelectual capital reporting: case study: The group of eight", Corporate Ownership \& Control, vol. 11, issue 4, pp. 288- 295.

Kaziliünas, Adolfas (2012), "The knowledge management process for implementing quality improvement programs", Informacijos Mokslai/Information Sciences, vol. 62, pp. 97-108.

Kenway, Jane y Johannah Fahey (2014), "Staying ahead of the game: the globalising practices of elite schools", Globalization, Societies and Education, vol. 12, issue 2, pp. 177-195.

Konno, Noboru, Ikujiro Nonaka y Jay Ogilvy (2014 a),
"The mind of the scenario thinker", World Futures, The Journal of New Paradigm Research, vol. 70, issue 1, pp. 44-51. Konno, Noboru, Ikujiro Nonaka y Jay Ogilvy (2014 b), "Scenario planning: the basics", World Futures, The fournal of New Paradigm Research, vol. 70, issue 1, pp. 28-43.

Konno, Noboru, Ikujiro Nonaka y Jay Ogilvy (2014 c), "Virtue-based management", World Futures, The Journal of New Paradigm Research, vol. 70, issue 1, pp. 19-27.

Lupton, Nathaniel y Paul Beamish (2014), "Organizational structure and knowledge-practice diffusion in the MNC", Journal of Knowledge Management, vol. 18, issue 4, pp. 710-727.

Markova, Gergana y Cameron Ford (2011), "Is money the panacea? Rewards for knowledge workers", International Fournal of Productivity and Performance Management, vol. 60, issue 8, pp. 813-823.

Martín de Castro, Gregorio (2014), "Intellectual capital and the firm: some remaining questions and prospects", Knowledge Management Research \& Practice, vol. 12, issue 3, pp. 239-245.

Mercado, Patricia, Mónica Sánchez y Yesenia Sánchez (2013), "Exploración del capital intelectual en instituciones públicas de educación superior. Un estudio comparativo entre dos universidades mexicanas", XIV Asamblea General de la Asociación Latinoamericana de Escuelas de Contaduría y Administración (ALAFEC), Panamá, 21-24 de octubre.

Mercado-Salgado, Patricia, Pedro Gil-Monte y Maria del Rosario Demuner-Flores (2014), "Development and initial validation of a survey for intellectual capital in universities", Proceedings of the European Conference on Knowledge Management, pp. 650-658.

Misiūnaitė-Bačiauskiené, Egle y Palmira Jucevičienè (2014), "Universiteto katedros studijų veiklos žinių valdymo proceso raiška", Pedagogika/Pedagogy, issue 115, pp. 8-24.

Mochales, Gerardo (2014), "Modelo explicativo de la responsabilidad social corporativa estratégica", Tesis de doctorado en Economía, Madrid, Universidad Complutense de Madrid.

Moreno-Brid, Juan Carlos y Pablo Ruiz-Nápoles (2009), La educación superior y el desarrollo económico en América Latina, México, Comisión Económica para América 
Latina y el Caribe (CEPAL)-ONU, Sede subregional en México (Serie estudios y perspectivas, 106).

Najim A., Najim, Mohamed Al-Naimi y Loay Alnaji (2012), "Impact of intellectual capital on realizing university goals in a sample of Jordanian universities", European Fournal of Business and Management, vol. 4, issue 14, pp. 153-162.

Nava-Rogel, Rosa María y Patricia Mercado-Salgado (2011), "Análisis de trayectoria del capital intelectual en una universidad pública mexicana", Revista Electrónica de Investigación Educativa, vol. 13, número 2, pp. 166-187.

Nava, Rosa, Patricia Mercado y María del Rosario Demuner (2012), "Indicadores de capital intelectual para instituciones públicas de educación superior", en Patricia Mercado Salgado, Daniel Arturo Cernas Ortíz (coords.), El capital intelectual en las organizaciones: una agenda de investigación, México, Universidad Autónoma del Estado de México, pp. 179-203.

Nonaka, Ikujiro y Hirotaka Takeuchi (1995), The Knowledge Creating Company, Nueva York, Oxford University Press.

Norma Internacional de Contabilidad (NIC) 38 (2013), Activos intangibles, International Financial Reporting Standards (IFRS), <http://www.ifrs.org/IFRSs / IFRStechnicalsummaries/Documents/Spanish\%20 Web\%20Summaries\%202013/IAS\%2038.pdf> [Consulta: abril de 2016].

Ordorika, Imanol (2014), "Sistema de información para la evaluación de la educación superior en México", Revista de la Educación Superior, vol. 43, número 171, pp. 5-7.

Ramírez, Yolanda, Jesús Santos y Ángel Tejada (2012), "Demanda de información sobre capital intelectual en las universidades públicas españolas", Cuadernos de Gestión, vol. 12, número 1, pp. 83-106.

Ramírez Yolanda, Ángel Tejada y Silvia Gordillo (2013), "Recognition of intellectual capital importance in the university sector", International fournal of Business and Social Research, vol. 3, issue 4, pp. 27-41.

Ramírez, Yolanda, Angel Tejada y Agustín Baidez (2013), "Proposal of indicators for reporting on intellectual capital in universities", Proceedings of the International Conference on Intellectual Capital, Washington, DC, pp. 355-366.
Ramírez Yolanda y Silvia Gordillo (2014), "Recognition and measurement of intellectual capital in Spanish universities", Journal of Intellectual Capital, vol. 15, issue 1, pp. 173-188.

Salleh, Kalsom (2014), "Learning organization and knowledge management: transfer process of tacit knowledge in public university for academic excellence", Proceedings of The International Conference on Intellectual Capital, Knowledge Management \& Organizational Learning, Sydney, Australia, pp. 347-353.

Sánchez, Paloma, Rocío Castrillo y Susana Elena (2006), "Intellectual capital management and reporting in universities", International Conference on Science, Technology and Innovation Indicators. History and New Perspectives, Lugano, pp. 15-17 noviembre.

Skjong, Rolf y Benedikte Wentworth (2000), Expert judgement and risk perception, <http://research.dnv.com/ skj/Papers/SkjWen.pdf> [Consulta: marzo de 2016].

United Nations (2005), Understanding knowledge societies in twenty questions and answers with the index of knowledge societies, Nueva York, United Nation Press.

Vaca, Byron (2015), "La comunicación en entornos virtuales $2 \mathrm{~d}$ y $3 \mathrm{~d}$. Un análisis con estudiantes del grado de ingeniería", Tesis doctoral, España, Universitat Rovira I Virgil.

Von Krogh, Georg, Ikujiro Nonaka y Lise Rechsteiner (2012), "Leadership in organizational knowledge creation: a review and framework", fournal of Management Studies, vol. 49, issue 1, pp. 240-277.

Wardini, Amalia (2015), "The IC practice of human capital in a university: an experience from Indonesia", Proceedings of the European Conference on Intellectual Capital, Cartagena, Murcia, España, pp. 474-483.

Wigmore-Álvarez, Ambery Mercedes Ruiz-Lozano (2014), "The United Nations Global Compact Progress Reports as management control instruments for social responsibility at Spanish universities", SAGE Open, April-June, pp. 1-12.

World Bank/International Bank for Reconstruction and Development (2013), World Development Indicators 2013, Washington, DC, World Bank. 
Gestión del conocimiento en la universidad: cuestionario para la evaluación institucional

\section{Anexo 1. Cuestionario de evaluación de la gestión del conocimiento universitario (CEGCU)}

Instrucción: Señor/a encuestado/a se solicita contestar de manera objetiva a las preguntas planteadas, que apoyarán la consecución de la investigación: "Modelo para la evaluación de la gestión del conocimiento universitario", que aporte al desarrollo de procesos de gestión de la universidad.

\begin{tabular}{|c|c|c|}
\hline \multicolumn{3}{|l|}{ 1. Información general } \\
\hline \multicolumn{3}{|l|}{ Datos de la Organización: } \\
\hline \multicolumn{2}{|l|}{ Nombre universidad: } & Número de docentes de la carrera: \\
\hline & & Menos de 50 \\
\hline \multicolumn{2}{|l|}{ Facultad: } & Entre 50 y $100 \bigcirc$ \\
\hline & & Más de 100 \\
\hline \multicolumn{2}{|l|}{ Carrera:- } & \\
\hline \multicolumn{3}{|l|}{ Datos del encuestado: } \\
\hline Género & Años de trabajo en la organización: & Nivel del cargo: \\
\hline$M O$ & Menos de $5 \quad O$ & Director/a de carrera $\bigcirc$ \\
\hline $\mathrm{F} \quad \mathrm{O}$ & Entre 5 y 10 & \\
\hline Innovación y creatividad & Entre 11 y $15 \quad O$ & \\
\hline Capital estructural & Entre 16 y 20 & \\
\hline Infraestructura física, sistemas informáticos & Más de 20 & \\
\hline
\end{tabular}

Sección 1. Gestión del conocimiento universitario (GC):

Nota: "La gestión del conocimiento, es el proceso que realizan las personas que integran la universidad para el desarrollo de productos académicos" La escala de valores de respuesta corresponde a:

\begin{tabular}{|l|l|}
\hline Nivel & Descripción \\
\hline 1 & Los procesos son desorganizados. \\
\hline 2 & Los procesos siguen un patrón regular. \\
\hline 3 & Los procesos están estandarizados. \\
\hline 4 & El proceso está integrado en la universidad y es monitoreado. \\
\hline 5 & El proceso se ha depurado hasta un nivel de mejor práctica. \\
\hline
\end{tabular}

\section{Marque sólo una opción:}

\begin{tabular}{|c|c|c|}
\hline ID & Procesos de gestión del conocimiento en la universidad & Escala \\
\hline \multicolumn{3}{|c|}{ 2. Creación de conocimiento en la universidad } \\
\hline CC1 & La universidad recolecta información de los docentes. & 12345 \\
\hline $\mathrm{CC} 2$ & La organización universitaria obtiene información de los estudiantes. & 12345 \\
\hline $\mathrm{CC} 3$ & Los archivos y las bases de datos proporcionan información necesaria a los integrantes de la organización. & 12345 \\
\hline
\end{tabular}




\begin{tabular}{|c|c|c|}
\hline CC4 & Los docentes de la universidad rompen las concepciones tradicionales. & 12345 \\
\hline CC5 & Los directivos de la organización realizan reuniones periódicas para informar los avances. & 12345 \\
\hline CC6 & La universidad posee políticas de compensación para el intercambio de conocimientos. & 12345 \\
\hline $\mathrm{CC} 7$ & Los informes escritos son distribuidos en la universidad. & 12345 \\
\hline $\mathrm{CC} 8$ & La información formal es compartida. & 12345 \\
\hline CC9 & La información informal es compartida. & 12345 \\
\hline CC10 & Los sistemas informáticos permiten compartir la información. & 12345 \\
\hline CC11 & La información necesaria puede obtenerse a partir de los archivos y las bases de datos. & 12345 \\
\hline $\mathrm{CC} 12$ & Los directivos concuerdan en que la información beneficia a la universidad. & 12345 \\
\hline $\mathrm{CC} 13$ & La universidad descarta la información obsoleta. & 12345 \\
\hline CC14 & La organización universitaria posee un orden para el desempeño de funciones. & 12345 \\
\hline \multicolumn{3}{|c|}{ 3. Transferencia y almacenamiento de conocimiento } \\
\hline TA1 & Los docentes monopolizan el conocimiento como fuente de poder. & 12345 \\
\hline TA2 & La rotación de docentes implica una pérdida de conocimiento. & 12345 \\
\hline TA3 & Las propuestas de los integrantes de la universidad se recogen con procedimientos. & 12345 \\
\hline TA4 & Las bases de datos almacenan experiencias y conocimientos. & 12345 \\
\hline TA5 & La universidad posee mecanismos formales para compartir las mejores prácticas. & 12345 \\
\hline TA6 & La organización universitaria conserva procedimientos para la distribución de las propuestas. & 12345 \\
\hline TA7 & El acceso a bases de datos se produce a través de la red de ordenadores. & 12345 \\
\hline TA8 & La organización aprende o adquiere conocimiento nuevo e importante. & 12345 \\
\hline TA9 & Los miembros de la universidad aprenden conocimientos o habilidades críticas. & 12345 \\
\hline TA10 & La universidad mejora por los nuevos conocimientos adquiridos. & 12345 \\
\hline TA11 & La universidad es una organización de aprendizaje. & 12345 \\
\hline \multicolumn{3}{|c|}{ 4. Aplicación y uso de conocimiento } \\
\hline $\mathrm{AU} 1$ & Los directivos de la organización universitaria promueven el trabajo en equipo. & 12345 \\
\hline $\mathrm{AU} 2$ & Los grupos de trabajo usan la información en la universidad. & 12345 \\
\hline $\mathrm{AU} 3$ & La universidad resuelve los problemas a través del trabajo en equipo. & 12345 \\
\hline AU4 & En la universidad los equipos de trabajo proponen soluciones innovadoras. & 12345 \\
\hline AU5 & La universidad adopta las recomendaciones de los grupos que la integran. & 12345 \\
\hline AU6 & Los docentes de la universidad redefinen la estrategia de la organización. & 12345 \\
\hline AU7 & Existe autocontrol de los docentes, y son responsables de su trabajo. & 12345 \\
\hline AU8 & Los docentes de la organización universitaria muestran autonomía en la toma de decisiones. & 12345 \\
\hline AU9 & Las sugerencias de los docentes se incorporan a la universidad. & 12345 \\
\hline
\end{tabular}


Gestión del conocimiento en la universidad: cuestionario para la evaluación institucional

\begin{tabular}{|c|c|c|}
\hline AU10 & La universidad establece alianzas y redes externas. & 12345 \\
\hline AU11 & La organización universitaria realiza acuerdos de cooperación para la promoción del conocimiento. & 12345 \\
\hline $\mathrm{A} \cup 12$ & Las sugerencias de los estudiantes se incorporan a la universidad. & 12345 \\
\hline $\mathrm{A} \cup 13$ & La organización conserva mecanismos para promover la creación de conocimiento. & 12345 \\
\hline AU14 & La universidad capacita y orienta a los docentes. & 12345 \\
\hline $\mathrm{A} \cup 15$ & Las bases de datos con información de los organismos relacionados se actualizan continuamente. & 12345 \\
\hline AU16 & Los procesos organizacionales de la universidad se documentan. & 12345 \\
\hline
\end{tabular}

\section{Sección 2. Capital intelectual universitario $(\mathrm{Cl})$}

Instrucción: En la presente sección, manifieste la cantidad de productos de conocimiento que la entidad bajo su dirección obtuvo durante el período enero - diciembre.

Nota: "El capital intelectual es todo producto académico que puede identificarse y cuantificarse".

\begin{tabular}{|c|c|c|}
\hline ID & Productos académicos & Cantidad \\
\hline \multicolumn{3}{|c|}{ 5. Capital humano } \\
\hline $\mathrm{CH} 1$ & Patentes activas a nivel nacional propiedad de la universidad. & \\
\hline $\mathrm{CH} 2$ & Derechos de propiedad intelectual conjuntos de profesores universitarios y empleados de la empresa. & \\
\hline $\mathrm{CH} 3$ & Derechos de autor generados. & \\
\hline $\mathrm{CH} 4$ & Publicaciones científicas individuales. & \\
\hline $\mathrm{CH} 5$ & Libros. & \\
\hline $\mathrm{CH} 6$ & Capítulos de libros. & \\
\hline $\mathrm{CH} 7$ & Visibilidad del sitio web de la universidad. & \\
\hline $\mathrm{CH} 8$ & Memorias de las participaciones de investigadores en conferencias y/o congresos internacionales. & \\
\hline $\mathrm{CH} 9$ & Investigadores visitantes internacionales (duración mínima 1 semana). & \\
\hline $\mathrm{CH} 10$ & Estancias de investigación en el extranjero. & \\
\hline $\mathrm{CH} 11$ & Memorias de conferencias realizadas por la universidad con relación al aprendizaje intercultural. & \\
\hline $\mathrm{CH} 12$ & Proyectos internacionales con socios de cooperación internacional. & \\
\hline $\mathrm{CH} 13$ & Investigadores que participan en proyectos de investigación con socios de cooperación internacional. & \\
\hline $\mathrm{CH} 14$ & Patentes internacionales. & \\
\hline $\mathrm{CH} 15$ & Autores altamente citados ( $\mathrm{HiCi})$ según Thomson Reuters. & \\
\hline $\mathrm{CH} 16$ & Número de citas internacionales por documento. & \\
\hline \multicolumn{3}{|c|}{ 6. Capital estructural } \\
\hline CE1 & Manuales de procedimientos. & \\
\hline CE2 & Plan estratégico de investigación (PEI). & \\
\hline CE3 & Mecanismos para evaluar el PEl. & \\
\hline CE4 & Revistas electrónicas indexadas. & \\
\hline
\end{tabular}


Gestión del conocimiento en la universidad: cuestionario para la evaluación institucional

Gabriela Cuadrado-Barreto / pp. 201-218

\begin{tabular}{|c|c|c|}
\hline CE5 & Centros de investigación de la universidad que posean líneas de estudio de temas globales. & \\
\hline CE6 & Sedes de la universidad en el extranjero con el enfoque de estrategia internacional. & \\
\hline CE7 & Universidades extranjeras que poseen sede en su universidad (oficinas internacionales). & \\
\hline CE8 & Bases de datos y estadísticas sobre la actividad internacional a nivel institucional. & \\
\hline CE9 & Programas de grado conjunto o doble con otras universidades. & \\
\hline CE10 & Políticas y estrategias para la internacionalización. & \\
\hline CE11 & Programas de internacionalización. & \\
\hline CE12 & Plan operativo de internacionalización. & \\
\hline \multicolumn{3}{|c|}{ 7. Capital relacional } \\
\hline CR1 & Acuerdos con empresas y organizaciones privadas y no gubernamentales. & \\
\hline CR2 & Acuerdos y convenios con organismos públicos. & \\
\hline CR3 & Copublicaciones internacionales. & \\
\hline CR4 & Citaciones a la universidad. & \\
\hline CR5 & Concursos ganados por la organización universitaria. & \\
\hline CR6 & Contratos con la industria para capacitación. & \\
\hline CR7 & Contratos con la industria para investigación y desarrollo. & \\
\hline CR8 & Redes de investigación interna y externa a la universidad. & \\
\hline CR9 & Publicaciones con coautores nacionales. & \\
\hline CR10 & Redes académicas internas y externas a la universidad. & \\
\hline CR11 & Convenios con otras universidades. & \\
\hline
\end{tabular}

\section{Cómo citar este artículo:}

Cuadrado-Barreto, Gabriela (2020), "Gestión del conocimiento en la universidad: cuestionario para la evaluación institucional", Revista Iberoamericana de Educación Superior (RIES), vol. XI, núm. 30, pp. 201-218, https://doi.org/10.22201/iisue.20072872e.2020.30.596 [Consulta: fecha de última consulta]. 